\title{
Graphene-polymer multilayer heterostructure for terahertz metamaterials
}

Zaiquan Xu, Caiyun Chen, Steve Qing Yang Wu, Bing Wang, Jinghua Teng, et al.

Zaiquan Xu, Caiyun Chen, Steve Qing Yang Wu, Bing Wang, Jinghua Teng, Chao Zheng, Qiaoliang Bao, "Graphene-polymer multilayer heterostructure for terahertz metamaterials," Proc. SPIE 8923, Micro/Nano Materials, Devices, and Systems, 89230C (7 December 2013); doi: 10.1117/12.2049533

SPIE Event: SPIE Micro+Nano Materials, Devices, and Applications, 2013, Melbourne, Victoria, Australia 


\title{
Graphene-polymer Multilayer Heterostructure for Terahertz Metamaterials
}

\author{
Zaiquan Xu, ${ }^{\mathrm{a}}$ Caiyun Chen, ${ }^{\mathrm{b}}$ Steve Qing Yang Wu, ${ }^{\mathrm{c}}$ Bing Wang, ${ }^{\mathrm{c},}{ }^{*}$ Jinghua Teng, ${ }^{\mathrm{c}}$ Chao Zhang, ${ }^{\mathrm{d}}$ Qiaoliang \\ Bao, ${ }^{\mathrm{a}, *}$
}

${ }^{a}$ Department of Materials Engineering, Monash University, Wellington Road, Clayton, Victoria, 3800, Australia

${ }^{\mathrm{b}}$ Institute of Functional Nano \& Soft Materials (FUNSOM), Soochow University, Suzhou, Jiangsu 215123, China

${ }^{\mathrm{c}}$ Institute of Materials Research and Engineering, Agency for Science, Technology and Research (A*STAR), 3 Research Link, Singapore 117602

${ }^{\mathrm{d}}$ School of Engineering Physics, University of Wollongong, New South Wales 2552, Australia

*Correspondence: wangb@imre.a-star.edu.sg; qiaoliang.bao@monash.edu

\begin{abstract}
The optical response and plasmon coupling between graphene sheets for graphene/polymer multilayer heterostructures with controlled separation were systematically investigated. Anomalous transmission of light was experimentally observed in mid-infrared range. The position of the broad passband in the transmission spectra was observed to red-shift with the increase of the number of layers.
\end{abstract}

\section{Introduction}

The surface plasmon in graphene has attracted increasing research interests because of many desirable properties such as strong confinement and low loss. ${ }^{1-3}$ The wavelength of graphene plasmons was experimentally observed at the order of 200 nanometres, more than 40 times smaller than the wavelength of illumination which is in the terahertz (THz) regime. ${ }^{4} 5$ Patterning graphene into micro-ribbons ${ }^{6}$ or photonic-crystal-like structures ${ }^{7}$ could induce plasmon resonance in $\mathrm{THz}$ range, which affords a new type of THz metamaterials. Graphene plasmonics is very promising to make transformation optics ${ }^{8}$ due to its tunability via chemical modification or electrical gating. The activities of surface plasmons in graphene multilayers can be controlled more flexibly than graphene monolayers because of the vertical interlayer coupling or in-plane lateral coupling. It has been theoretically predicted that the plasmon resonance can be controlled by the coupling effect among adjacent layers, whereas less attention has been paid to the experimental demonstration. ${ }^{9,10}$

Here we fabricate graphene/polymer multilayer heterostructure and systematically investigate the optical response as well as plasmon coupling between graphene sheets. Anomalous transmission of light was experimentally observed in $\mathrm{THz}$ range. The graphene/polymer multilayers could be a promising platform to filter infrared and terahertz waves and to tailor the propagation and diffraction of light along the graphene sheets deeply below the sub-diffraction-limit. 


\section{Experiments}

Large area monolayer graphene films used in the work were grown by chemical vapour deposition (CVD) on $25 \mu \mathrm{m}$ thick $\mathrm{Cu}$ foil (Alfa Aesar AA13382RG). Poly(methyl methacrylate) (PMMA) was spin-coated on top of the graphene, the resulting thickness was characterized by variable angle spectrometric ellipsometer (VASE). The copper used to grow graphene was etched away and monolayer graphene-PMMA (G-PMMA) heterostructure was wet-transferred onto intrinsic silicon substrates after doping with $\mathrm{HNO}_{3}$. Multilayer heterostructure was formed by repeatedly transferring one layer of G-PMMA on top of the other, as shown in Fig. 1. THz transmittance measurements were carried out on Bruker Vertex 80V Fourier Transform Infrared (FTIR) with a mercury lamp as THz source and DTGS as THz detector.

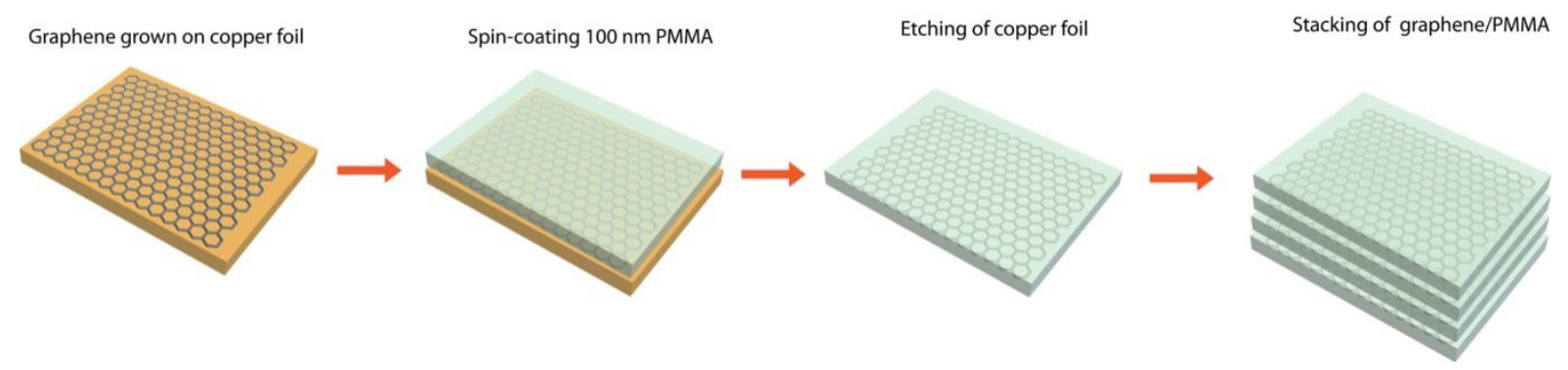

Figure 1. Schematic diagram of fabrication procedure for graphene/PMMA heterostructures.

\section{Experimental and simulation results}

The Raman spectra and images were measured on WITEC Alpha 300 confocal micro-Raman system equipped with a $532 \mathrm{~nm}$ laser source and $100 \times$ objective lens. The representative Raman spectrum from monolayer graphene transferred onto intrinsic silicon is shown in Fig. 2. It is found that the Raman $2 \mathrm{D}$ band is much stronger than $G$ band with a $2 \mathrm{D} / \mathrm{G}$ ratio of 1.5 , indicating the nature of one atomic layer. The $\mathrm{G}$ and $2 \mathrm{D}$ band can be fitted well by single Lorentzian peak with full-width at half-maximum (FWHM) of 26 and $38 \mathrm{~cm}-1$ respectively, which suggested that the graphene used was of high crystalline quality. These are key Raman characteristics for monolayer graphene. 


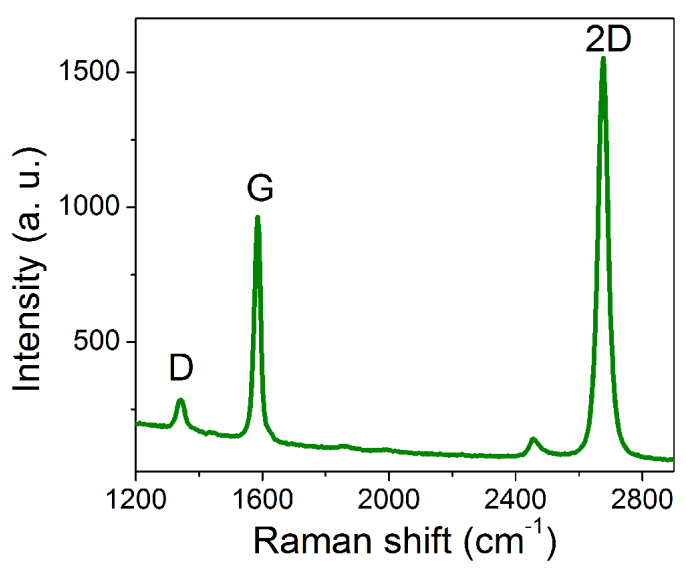

Figure 2. Raman spectrum of monolayer graphene.

The extinction spectra measured on as-prepared PMMA/Graphene (PMMA/G) heterostructures with one, two and three graphene layers are shown in Fig. 3a and Fig. 3b. It is found that the number of graphene layers plays an important role to determine the extinction ratio. The introduction of monolayer graphene between the polymer and intrinsic silicon (i-Si) leads to an increase of the extinction ratio up to $22.5 \%$ at $2.55 \mathrm{THz}$ due to free carrier absorption. ${ }^{7}$ The extinction ratio increases with the number of graphene sheets, i.e., an extinction ratio of $55.6 \%$ is achieved for a graphene/polymer heterostructure with three graphene layers.

The transmission properties of multilayer graphene sheets and in particular, the influence of the number of graphene sheets are studied as well, as shown in Fig. 3c. A passband can be observed for the samples with two, three, four, five graphene sheets. It is very interesting to find that the peaks of the passband evolve with the number of graphene sheets, i.e., the lager the number of graphene sheets, the sharper the edges of the passband and the lower the transmission peak intensity. The transmission peak tends to shift towards longer wavelength for larger number of graphene sheets, due to the lateral boundary surface plasmon polarition (SPP) coupling between graphene sheets. ${ }^{10}$
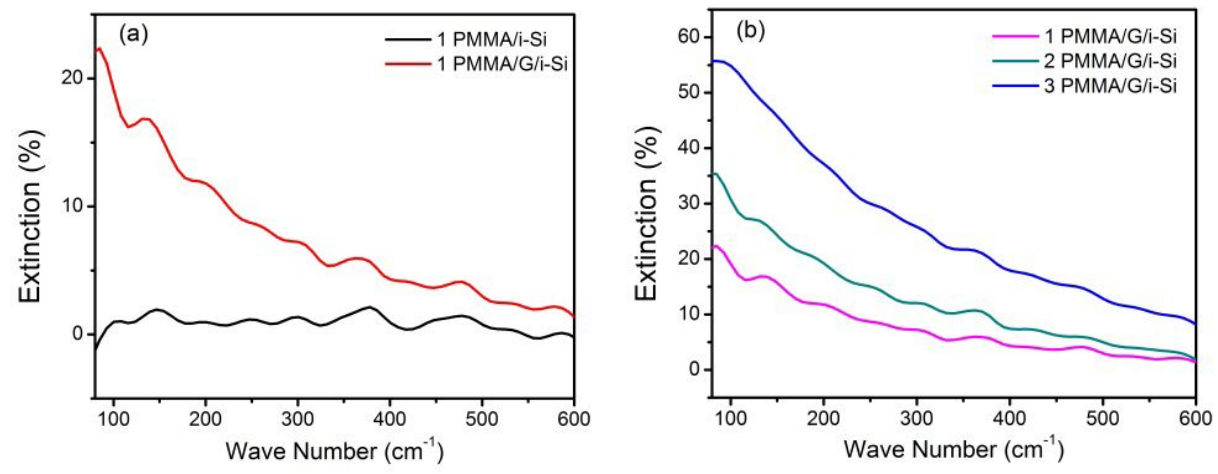


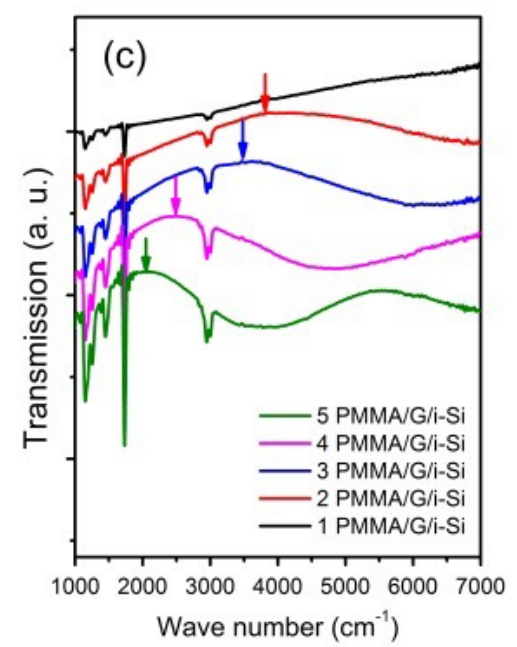

Figure 3. (a) The extinction spectra of monolayer PMMA/G stack and reference sample (PMMA only on intrinsic silicon substrate). Inset shows the schematic of PMMA/G stack. (b) The extinction spectra of the PMMA/G stacks with different number of layers; (c) The transmission spectra as a function of the number of the doped graphene sheets.

\section{Conclusion and outlook}

In summary, we have studied light transmitting properties of the graphene multilayers composed of arbitrary layers of graphene sheets. The extinction co-efficiency resulting from monolayer graphene/polymer stack due to the free carrier absorption is $\sim 22.5 \%$ and it increases with the number of layers. A broad passband was observed in the transmission spectrum and it can be tuned by the number of layers. Future efforts will be put on tuning the broadband transmission by electrically gating the heterostructures.

\section{Reference}

1. Bao, Q. L.; Loh, K. P. Graphene Photonics, Plasmonics, and Broadband Optoelectronic Devices. ACS Nano 2012, 6, 3677-3694.

2. Grigorenko, A.; Polini, M.; Novoselov, K. Graphene Plasmonics. Nature Photonics 2012, 6, 749-758.

3. Koppens, F. H.; Chang, D. E.; García de Abajo, F. J. Graphene Plasmonics: A Platform for Strong Light-Matter Interactions. Nano letters 2011, 11, 3370-3377.

4. Chen, J.; Badioli, M.; Alonso-González, P.; Thongrattanasiri, S.; Huth, F.; Osmond, J.; Spasenović, M.; Centeno, A.; Pesquera, A.; Godignon, P. Optical Nano-Imaging of Gate-Tunable Graphene Plasmons. Nature 487, 77-81 2012.

5. Fei, Z.; Rodin, A.; Andreev, G.; Bao, W.; McLeod, A.; Wagner, M.; Zhang, L.; Zhao, Z.; Thiemens, M.; Dominguez, G. Gate-Tuning of Graphene Plasmons Revealed by Infrared Nano-Imaging. Nature 487, $82-852012$.

6. Ju, L.; Geng, B.; Horng, J.; Girit, C.; Martin, M.; Hao, Z.; Bechtel, H. A.; Liang, X.; Zettl, A.; Shen, Y. R. 
Graphene Plasmonics for Tunable Terahertz Metamaterials. Nature Nanotechnology 2011, 6, 630-634.

7. Yan, H.; Li, X.; Chandra, B.; Tulevski, G.; Wu, Y.; Freitag, M.; Zhu, W.; Avouris, P.; Xia, F. Tunable Infrared Plasmonic Devices Using Graphene/Insulator Stacks. Nature Nanotechnology 2012, 7, 330-334.

8. Vakil, A.; Engheta, N. Transformation Optics Using Graphene. Science 2011, 332, 1291-1294.

9. Wang, B.; Zhang, X.; García-Vidal, F. J.; Yuan, X.; Teng, J. Strong Coupling of Surface Plasmon Polaritons in Monolayer Graphene Sheet Arrays. Physical Review Letters 2012, 109, 073901.

10. Wang, B.; Zhang, X.; Yuan, X.; Teng, J. Optical Coupling of Surface Plasmons between Graphene Sheets. Applied Physics Letters 2012, 100, 131111-4. 